\section{(O) OPEN ACCESS}

\title{
Loss to specialist follow-up in congenital heart disease; out of sight, out of mind
}

\author{
Jo Wray, Alessandra Frigiola, Catherine Bull, Adult Congenital Heart disease Research \\ Network (ACoRN)
}

Department of Cardiology, Great Ormond Street Hospital, London, UK

\section{Correspondence to} Dr Jo Wray Department of Cardiology, Great Ormond Street Hospital NHS Foundation Trust, London WC1N 3JH, UK; jo.wray@gosh.nhs.uk

\section{ACoRN contact: Dr Mark} Turner, Consultant Cardiologist, The Bristol Heart Institute Hospital, Bristol, BS2 $8 \mathrm{HW}, \mathrm{UK}$.

Accepted 25 September 2012 Published Online First 13 February 2013

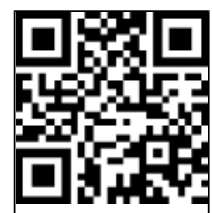
Open Access Scan to access mor free content

\section{SLinked}

- http://dx.doi.org/10.1136/ heartjnl-2012-303121

\begin{abstract}
Objective To evaluate the scale and clinical importance of loss to follow-up of past patients with serious congenital heart disease, using a common malformation as an example. To better understand the antecedents of loss to specialist follow-up and patients attitudes to returning.

Design Cohort study using NHS number functionality.
\end{abstract} Content and thematic analysis of telephone interviews of subset contacted after loss to follow-up.

Patients, intervention and setting Longitudinal follow-up of complete consecutive list of all 1085 UK patients with repair of tetralogy of Fallot from single institution 1964-2009.

Main outcome measures Survival, freedom from late pulmonary valve replacement, loss to specialist followup, shortfall in late surgical revisions related to loss to follow-up. Patients' narrative about loss to follow-up.

Results $216(24 \%)$ of patients known to be currently alive appear not to be registered with specialist clinics; some are seen in general cardiology clinics. Their median age is 32 years and median duration of loss to follow-up is 22 years; most had been lost before Adult Congenital services had been consolidated in their present form. $48 \%$ of the late deaths to date have occurred in patients not under specialist follow-up. None of those lost to specialist follow-up has had secondary pulmonary valve replacement while 188 patients under specialist care have. Patients lost to specialist follow-up who were contacted by telephone had no knowledge of its availability.

Conclusions Loss to specialist follow-up, typically originating many years ago, impacts patient management.

\section{INTRODUCTION}

Open heart surgery for congenital heart disease began in the 1960s in the UK. Its progressive success has meant that since around 2002, adult survivors of surgery outnumber children with congenital heart disease in the UK. ${ }^{1}$ The same document estimated that there are around 130500 adults with 'moderate' or 'complex' congenital heart disease alive in England and Wales today; almost all are survivors of surgery they had as young children. For many complex congenital heart diseases, residual haemodynamic problems can result in late heart failure, reduced exercise capacity, arrhythmias or sudden death and there are risks associated with pregnancy and non-cardiac surgery. ${ }^{3}$ Most premature deaths associated with congenital heart disease are now occurring in adults rather than children.

Tetralogy of Fallot is the commonest 'cyanotic' congenital heart disease. Palliative surgery for this malformation became available in the UK in the late $1950 \mathrm{~s},{ }^{4}$ and intracardiac repairs in the early 1960s. Without surgery, only 4\% of children born with tetralogy of Fallot would be alive at 15 years. ${ }^{5}$ The intracardiac repair is generally successful in closing the ventricular septal defect but most patients are left with an element of pulmonary obstruction or incompetence. Timely reintervention with insertion of a 'pulmonary' valve in the right ventricular outflow tract may help to prevent or delay late complications. While the exact indications for reintervention are not yet entirely clear, recommendations for pulmonary valve replacement (PVR) may be made on the basis of physiological parameters even if a patient has few symptoms. ${ }^{6}$ For patients after Fallot repair, current guidelines stipulate that patients be periodically reviewed by specialists accredited in Adult Congenital Heart Disease (ACHD) in designated centres, though shared-care networks allow for delivery of some care by cardiologists with competencies in ACHD. ${ }^{1}{ }^{2}$ However, ACHD provisions have been consolidated in their present form for less than 15 years and not all patients who left childhood services before this period have found their way to these services.

In this study, we aimed to use tetralogy of Fallot with its requirement for lifelong surveillance and possible adult reoperation to explore the implications of loss to follow-up of congenital heart disease. For a well-defined cohort, we aimed to establish the proportion of living patients not being followed to the specified standard and to estimate the impact of any shortfall in follow-up coverage on the incidence of secondary reoperations. We also wanted to conduct telephone interviews with patients believed to be lost to follow-up to understand their views on follow-up.

\section{METHODS}

We created a complete consecutive list of patients who had repair of uncomplicated tetralogy of Fallot at Great Ormond Street Hospital (GOSH) from the very first repair performed in February 1964 to January 2009; patients receiving an extracardiac conduit at their primary repair were excluded as this commits them to subsequent surgery. Patients from abroad were excluded as we have no control of their follow-up. The list was 
generated using several sources: the manually written surgical lists (patients operated in the 1960s and 1970s), scanned operation notes (patients from the 1970s and 1980s), and from various electronic databases used since then. Archived medical records were checked and early postoperative deaths and the dates of any childhood PVRs were determined. Current patients have National Health Service (NHS) numbers recorded within their childhood records, but this is not true for patients lost before these came into existence in 1996. Using the known dates of birth and childhood surnames, NHS numbers were linked to the patient records using batch tracing functions and manual searches. At around the age of 16, patients are currently discharged to the care of their nearest ACHD service; this process is systematic at present but historically it was much more variable. Given a list of NHS numbers and current names, addresses and dates of birth, collaborating specialist cardiologists from the Adult Congenital heart disease Research Network scanned their local databases for the listed patients to ascertain whether they had died under their care or were under active review or had been previously seen but then 'lost'; if they had undergone PVR they established the date of PVR when this had first occurred beyond childhood. Where the specialist centres, patients' families or the NHS core record alerted us to a late death, the date of death was documented. Patients were deemed 'lost to specialist follow-up' if they had not been seen within any specialist network in the past 3 years. Patients known to have relocated abroad or whose NHS numbers we could not establish were censored on the date last seen alive. Because PVR is only undertaken in specialist centres, live patients who had corresponding NHS numbers but were lost to specialist follow-up were assumed not to have had PVR.

\section{Telephone interviews}

The General Practitioners (GPs) of patients who had repaired tetralogy of Fallot at GOSH and were believed not to be under specialist follow-up were contacted to confirm that we had identified the correct patient. On receiving confirmation, patients were sent an explanatory letter asking them to contact us if they were prepared to receive a telephone call.

Patients were contacted by telephone at a time convenient to them. The telephone interview was semistructured and extensive notes (mostly verbatim quotes) were taken contemporaneously by the researcher (JW). Questions were asked concerning participants' current state of health and any interactions with health professionals, their recollection of any advice they had been given regarding their heart condition, use of support groups, knowledge about their condition, health experiences since the time of their surgery, attitudes to and experiences of follow-up and knowledge about current recommendations regarding follow-up of adult patients with congenital heart disease. Though it was made clear that the study did not aim to influence their follow-up arrangements, if participants subsequently wanted follow-up with specialist ACHD providers we contacted their GPs to request this.

\section{Statistical analysis}

Life table methodology, ${ }^{7}$ was employed to determine the cumulative incidence of PVR in surviving patients. To illustrate the impact of loss to follow-up on rates of PVR over time, two curves were generated. The first included all patients known from the NHS record to be alive, regardless of follow-up status and provides the 'actual' proportions over time. The second included patients only while they were actually under specialist care, the 'lost' patients being censored on the date last seen by a specialist centre, usually GOSH. SPSS V.18 was used.

\section{Qualitative analysis}

The first stage of the analysis of the telephone conversations involved familiarisation with the data by reading and re-reading the notes and quotes. Thematic analysis was used to generate initial codes from each interview and to identify themes across the dataset. ${ }^{8}$ Content analysis was used to determine the current arrangements for follow-up, access to support and requests for further information and/or referral to specialist ACHD services.

The project was approved by Research Ethics Committees within the National Research Ethics Service (NRES).

\section{RESULTS}

From February 1964 to January 2009, 1085 UK patients $(58 \%$ males) had repair of Fallot at GOSH, at a mean age of 3.5 \pm 3.6 years. Of 1085 , we have either known dates of death or NHS numbers and hence mortality status in all but 27 . Most of the 27 who have not been matched to their NHS numbers are adult women and a further 17 patients are known to have relocated abroad. 148 are known to have died to date. 100 died within 1 year of repair (24 of 106 patients (23\%) operated on in the 1960s, and 6 of 246 (2\%) operated in the 2000s). Of the subsequent deaths, 23/48 (48\%) appear not to have occurred while the patient was under specialist care, so any attribution of causes of late death for this cohort is unreliable.

Of the 893 known to be alive and living in the UK, 216 (24\%) appear not to have been seen in specialist centres including our own for more than 3 years (figure 1). The total of 216 includes 22 young people who have been lost from our own clinics for more than 3 years, while 194 are not attending ACHD clinics. The median current age of 'lost' patients is 32 years and median duration of loss to follow-up is 22 years.

A first PVR has been performed to date in 188 patients at a mean age of $20 \pm 13$ years, all necessarily in patients under specialist care. Figure 2 shows the cumulative proportion of live patients who have had PVR. Under the untested assumption that patients lost to follow-up lie in the same spectrum of primary anatomy and of incompleteness of surgical repair as those under active follow-up, the difference between these curves suggests that an additional 63 PVRs would have occurred, had the patients been followed by specialists.

\section{Telephone interviews}

Letters were sent to 176 GPs and as a consequence letters inviting a telephone call were sent to 100 patients; in six cases we were asked not to contact patients. One patient had been incorrectly tracked. Forty-two patients/carers of patients agreed to be contacted but five were not available. The 37 patients contacted by telephone included 23 (59\%) males with a median age current age of 44 years (range 9-57 years) and a median time since surgery of 37 years (range $8-47$ years).

Participants varied in terms of their current follow-up arrangements and in their requirements for changes to these arrangements (table 1). The majority of patients commented that 'they knew what they wanted to' about their condition, although few had had any contact with support groups or accessed information about their condition. A number of participants had not known how to access further support or information, as the following quote illustrates: 'I looked at the internet when I got your letter-I could spell tetralogy of Fallot then and look it up'. 


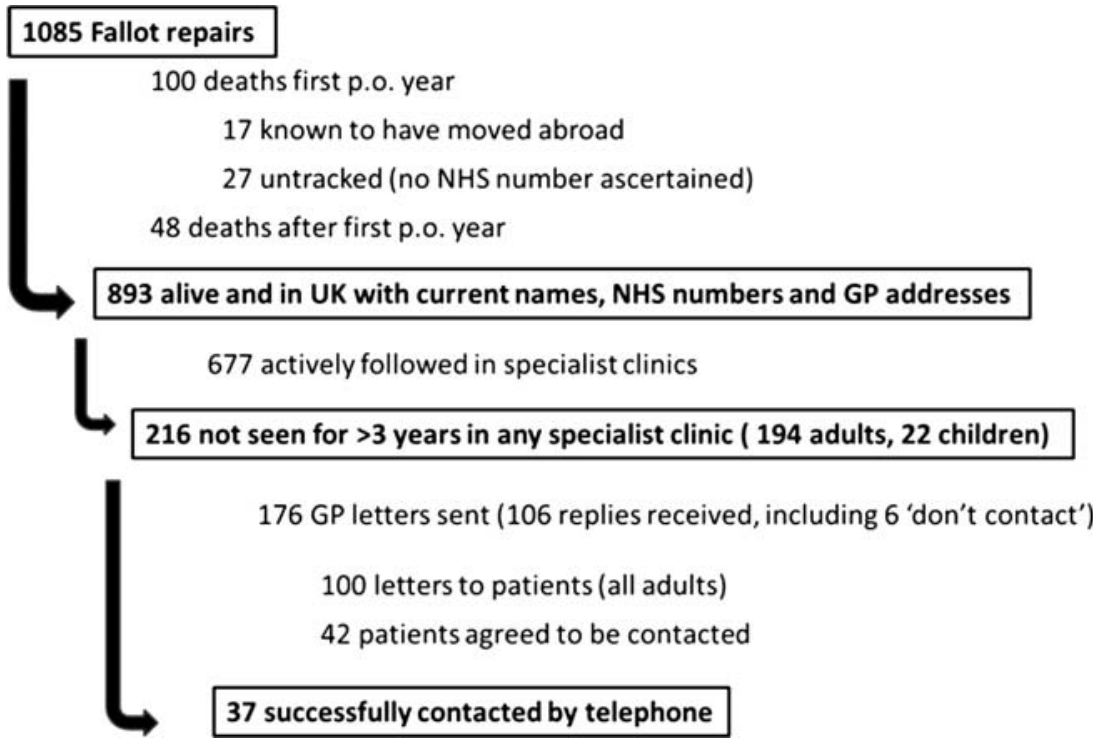

Figure 1 Flow diagram showing outcomes and background of patients contacted by telephone.

Themes from the qualitative analysis

Two main themes concerning attendance for follow-up emerged from the interviews: perceived responsibility and reassurance. Patients saw responsibility for maintaining follow-up as primarily the duty of health professionals (box 1) or participants saw it as their responsibility (box 2); within each of these there were a number of subthemes. Reassurance was described by many

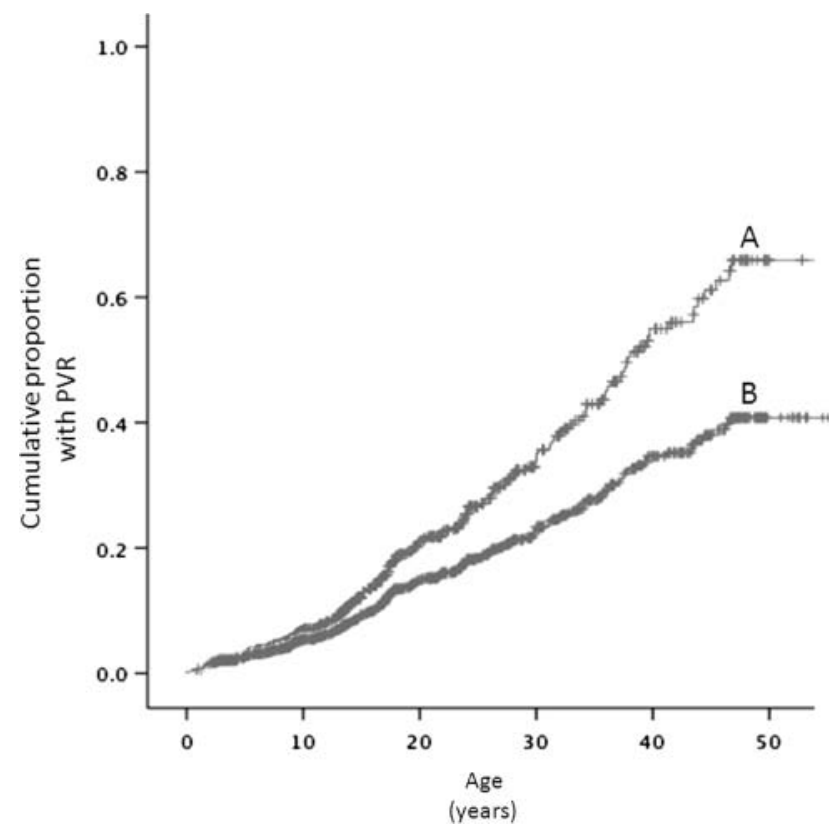

Figure 2 Cumulative proportion by age of patients having pulmonary valve replacement (PVR). The two curves represent the results obtained if (A) patients are only included while under active follow-up and (B) if all patients contribute in their lifetime whether or not they are under specialist follow-up. 'Lost' patients will not have had a PVR. Patients are censored if they died or moved abroad. In curve A, 'lost' patients are censored on the date last seen alive by a specialist hospital. participants-those receiving and not receiving follow-up-as the main benefit of follow-up (box 3).

\section{DISCUSSION}

This paper illustrates the clinical impact of loss to follow-up, often originating many years ago and before ACHD services were consolidated in their current form. Data from our cohort suggests that $38 \%$ of Fallot patients known to be alive and over the age of 30 are not being seen by ACHD specialist cardiologists and $48 \%$ of the late deaths recorded have occurred while not under such follow-up. Related to this there is a shortfall that is less easy to quantify in the incidence of late PVR, an operation typically undertaken as an investment for a patient's later quality of life.

This exercise, which is among the most comprehensive outcome studies to date, ${ }^{9}$ has been made possible by three resources: an early investment by one centre in maintaining good paper and electronic lists over decades, the functionality offered by the NHS number which has its own linkage to death certification data and the collaboration offered by specialists in the field of ACHD. Though individual specialist centres can document the number of patients each follows, the denominator-the total number of adult survivors of childhood operations including those who are not under follow-up-has not been available. The extent of loss to follow-up was explored in a somewhat

Table 1 Content analysis of adult responses $(n=37)$

Current follow-up arrangements

Not receiving any follow-up 15

Seen at local hospital by non-ACHD cardiologist/technician

Seen in adult services since left GOSH, then lapsed

Longest time without any heart related check-up

Aware of current recommendations regarding follow-up

Number of patients wanting referral for specialist follow-up

Number not receiving follow-up who explicitly did not want it

Requests for further written information/specific CHD websites

ACHD, Adult Congenital Heart Disease; $C H D$, congenital heart disease. 
Box 1 Perceived responsibility — health professionals

The input from professionals was discussed as an explanation for not attending for follow-up:

Perceived lack of interest or guidance

"I am not being followed-up because no-one has ever bothered..... Follow-up was never suggested before".

"It has never been up to me before-I have never not wanted to see someone but no-one told me".

"Follow-up is important but it is not taken seriously enough by some GPs".

Being led to believe follow-up was not necessary-they were "cured"

"I don't see anyone- I was signed off-that was it. I was told I would be fine"

"I was led to believe that it was all fixed".

"If it had been impressed on me that something could go wrong I would have made sure I had check-ups. Accurate perceptions are important".

"I assumed everything was OK because we didn't hear".

comparable study from Canada. ${ }^{10}$ This documented that of 643 patients born in 1983 and who had survived childhood with congenital heart disease, only $39 \%$ were receiving specialist follow-up after their 22nd birthday. Even among the most severe group, only $79 \%$ were periodically reviewed by specialists.

Like most repairs for congenital heart disease, Fallot surgery is not fully corrective with chronic pulmonary regurgitation being the most prevalent residual problem. Chronic pulmonary regurgitation is very well tolerated for many years, so a decision to discharge early patients from follow-up may have seemed reasonable. The exact physiological indications for PVR are not yet clear, but symptoms emerge almost imperceptibly in patients whose impairment is longstanding; objectively measured exercise capacity can be very poor in patients with good selfestimated capability. ${ }^{11}$ In principle, pulmonary regurgitation can be alleviated by insertion of a new pulmonary valve. Currently first pulmonary valve implants must be done surgically but the potential of percutaneous pulmonary valve implantation for Fallot survivors is encouraging. If PVR is to be done, ideally it will be undertaken electively when the patient is of adult size, as available valves do not grow with the patient. Leaving the operation too late prejudices right ventricular recovery and increases postoperative morbidity. ${ }^{6}$ Though transition to adult specialist services is more consistent in current practice, patients lost to follow-up before ACHD services emerged may not present again until late symptoms of cardiac decompensation occur.

Loss to specialist follow-up clearly has implications for the 'lost' patients themselves. We note that 23 patients were not known to specialist services at the time of their deaths. As only $3 \%$ of deaths in the general population occur in the age group $15-44,{ }^{12}$ we can infer that some of these deaths are likely to have related to their cardiac status and these deaths have not contributed to our understanding of this 'new' disease. Similarly we estimate that there is a significant shortfall in late PVRs related to loss to follow-up.

But in the context of 'new diseases' that emerge in the wake of evolving technologies, loss to follow-up also has implications for actively monitored patients and the doctors managing them. For instance, exact indications for PVR are not currently

\section{Box 2 Perceived responsibility: self}

Participants identified that their own behaviours and beliefs contributed to attending follow-up.

Leading a normal life and Fallot not part of that

"I moved around a lot-went to uni-never bothered to keep it connected".

"In one way I am glad that I had 10 years of blissful ignorance -I didn't see myself as damaged or in need of care".

Responsibility to others

"An extra check is better for me and helps others... You have a responsibility to people who have put you right".

"I now go for follow-up because of my partner and wanting to have a family".

Anxiety - can prevent or facilitate accessing follow-up care

"The hospital may have sent appointments and I didn't keep them. I am worried [about being followed-up]".

"I want to know what my heart is actually doing-am I fine? I want to know if there is anything wrong.... Why would you not go-it is your health. Things can change when you are older. Follow-up is really important-I would not miss it".

"I don't need follow-up-it is not my bag. What would they do? I hate needles...."

Ambivalence

"I don't think it did me any favours because I had to put on a form for an HGV license that I had been to the hospital and now I get hassle [because of it]. I wouldn't do it again-but they do keep an eye on me".

"If I had had to go back for follow-up it might have put questions in my mind...."

Denial

"I looked up a bit recently about TOF but ..... I want to pretend there is nothing wrong"

"I never think about it! I know that there are no guarantees in life-but that is with everything. I feel fine! I feel there is no need for follow-up"

"It was something that happened, it was sorted and I moved on".

"I am happy with how things are. I don't want any specialist follow-up".

\section{Awareness}

"I have never had any concerns. Your letter was the first time I had thought about it but there is no reason why I would not want follow-up".

"Now that I am aware I might well reconsider it".

\section{Box 3 Reassurance as the main benefit of follow-up}

Participants talked about the reassurance that follow-up could/ does provide

"It would be good to be seen by a specialist-reassuring".

"Follow-up helps, gives you confidence-they will spot anything that might be there".

"After all this time I expect to carry on as normal but with the reassurance of check-ups".

"I look forward to being checked out and I recommend that the system doesn't leave it so long to get in contact with its patients". 
established, ${ }^{6}$ but decision-making is inevitably only informed by the population under active follow-up. If the 'lost' patients were in a better clinical state than those seen in clinics, we might have a more optimistic view of the natural history of pulmonary regurgitation and delay surgery. If they were 'worse', we might bring forward recommendations for reoperation.

The telephone interviews have provided some insight into why patients have been lost to follow-up. It is evident that some patients not currently under follow-up want to be seen by specialist services but do not know how to access such services; the process of conducting the interviews has re-introduced half of those we spoke to who were not in specialist follow-up back into the system. Older patients in particular may have misperceptions about the need for follow-up and a lack of knowledge about potential problems that may arise. ${ }^{13}$

Anxiety and ambivalence may be keeping other patients out of follow-up. Others have identified the importance of normalising the clinical experience for adult patients with CHD while avoiding overmedicalising them. ${ }^{14}$ The importance of reassurance was a theme across all participant groups, irrespective of their follow-up arrangements, and ensuring that patients are aware of the benefits of regular check-ups as well as the potential problems which may arise. In the past decade, the responsibility of paediatric services to ensure continuity of care through transitioning to adult services has been operationalised, but patients also need to be empowered by those in the paediatric centre to share long-term responsibility for their own health and follow-up. As one participant said, 'Follow-up is like an MOT on a car; it takes one afternoon and if there is anything it will get picked up early rather than too late'.

Assuming the shortfall we have observed for tetralogy of Fallot is not unique, we anticipate that other lesions with few late symptoms—such as aortic coarctation-may be similarly impacted by loss to follow-up. For such patients, the challenges remain about if and how the specialist community should reach those not receiving any follow-up at all, as well as ensuring that those currently seen in non-specialist adult cardiology services receive optimum surveillance.

\section{Limitations}

Data linkage has been possible for all but 27/1085 patients; the proportions of these 27 patients who may have died, moved abroad, changed their names or whose original data were incorrect is unknown and would have a bearing on the estimates we provide. The cardiac status of the patients not under specialist follow-up may be better, worse or similar to those who are, though we have no particular indication that their original loss to follow-up was informative in this respect. Though we know that at least three early patients had catastrophic brain damage perioperatively and other adults (at least five) have Down's or Noonan's syndromes, ascertainment is too inconsistent to be able to explore whether or not such patients are underrepresented or over-represented among those lost to follow-up entirely or are seen in non-specialist cardiology clinics. We also have no data about the causes of about half the late deaths.

The research ethics committee dealing with the telephone contact stipulated that patients should not be contacted without the participation of their GPs and that patients should be left with the initiative concerning the phone calls. In the event, we received no response to $40 \%$ of the letters sent to GPs and of the patients approached with their GPs consent, only 42\% agreed to be contacted. We have observed elsewhere the requirement of a patient's involvement being contingent on their GP's cooperation has negatively impacted research findings in other contexts ${ }^{15}$ and here too the potential for bias from this shortfall makes it unrealistic for us to estimate the proportion of 'lost' patients who would like to return to follow-up. However, the insights provided by those who we did speak to have furthered our understanding of why patients are not being seen and, although not an aim of the study, the request for referral for specialist follow-up from a significant proportion of those we spoke to is a positive outcome of this work.

\section{Conclusions}

A significant number of patients living after Fallot surgery are not getting the follow-up they need. If the $25 \%$ of patients not in specialist follow-up documented here for tetralogy of Fallot is typical of 'moderate' congenital heart disease in general, 15000 patients in England and Wales may similarly be inadequately served. ${ }^{1}$ Patients with a clinical background of congenital heart diseasehowever well currently—should be encouraged back into specialist Adult Congenital cardiology clinics. ACHD is a new class of disease and complete datasets like this one are needed to delineate its natural history, inform appropriate and cost-effective follow-up programs and define treatment requirements.

Acknowledgements The Adult Congenital heart Research Network provided information about follow-up of patients in the cohort.

Contributors All authors contributed to the planning, conduct and reporting of the work described in the article. CB is responsible for the overall content as guarantor.

Funding This project was funded by the Great Ormond Street Hospital Children's' Charity.

Competing interests None.

Patient consent Obtained.

Ethics approval Institute of Child Health and Cambridge East.

Provenance and peer review Not commissioned; externally peer reviewed.

Data sharing statement Suitably anonymised data relating to the cohort will be available.

Reporting checklist This presentation conforms to the STROBE guidelines for cohort studies (checklist August 2012).

Open Access This is an Open Access article distributed in accordance with the Creative Commons Attribution Non Commercial (CC BY-NC 3.0) license, which permits others to distribute, remix, adapt, build upon this work non-commercially, and license their derivative works on different terms, provided the original work is properly cited and the use is non-commercial. See: http://creativecommons.org/ licenses/by-nc/3.0/

\section{REFERENCES}

1 A commissioning guide for services for young people and adults with congenital heart disease also known as Grown Ups with Congenital Heart Disease or GUCH). DH Vascular Programme Team; 2006, DH Publications http://www.dh.gov.uk/en/ Publicationsandstatistics/Publications/PublicationsPolicyAndGuidance/DH 4134608 (accessed 7 Jun 2011).

2 British Cardiac Society Working Party. Grown-up congenital heart (GUCH) disease: current needs and provision of service for adolescents and adults with congenital heart disease in the UK. Heart 2002;88(Suppl 1):i1-i14.

3 Bédard E, Shore DF, Gatzoulis MA. Adult congenital heart disease: a 2008 overview. Br Med Bull 2008:85:151-80

4 Blalock A, Taussig $H$. The surgical treatment of malformation of the heart in which there is pulmonary stenosis or pulmonary atresia. JAMA 1945;128:189-202.

5 Samanek M. Children with congenital heart disease: probability of natural survival. Pediatr Cardiol 1992;13:152-8.

6 Frigiola $A$, Tsang $V$, Bull $C$, et al. Biventricular response after pulmonary valve replacement for right ventricular outflow tract dysfunction. Circulation 2008;118: S182-90.

7 Kaplan EL, Meier P. Nonparametric estimation from incomplete observations. J Am Statist Assn 1958:53:457-81.

8 Braun V, Clarke V. Using thematic analysis in psychology. Qual Res Psychol 2006;3:77-101

9 Nollert G, Fischlein T, Bouterwek S, et al. Long-term survival in patients with repair of tetralogy of Fallot: 36 -year follow-up of 490 survivors of the first year after surgical repair. J Am Coll Cardiol 1997;30:1374-83. 
10 Mackie AS, Ionescu-Ittu R, Therrien J, et al. Children and adults with congenital heart disease lost to follow-up: who and when? Circulation 2009;120:302-9.

11 Gratz A, Hess J, Hager A. Self-estimated physical functioning poorly predicts actual exercise capacity in adolescents and adults with congenital heart disease. Eur Heart J 2009;30:497-504.

12 Aylin P, Dunnell K, Drever F. Trends in mortality of young adults aged 15-44 in England and Wales. Health Stat Q 1999:34-9.
13 Fox D, Devendra GP, Hart SA, et al. When 'blue babies' grow up: what you need to know about tetralogy of fallot. Cleveland Clin J Med 2010:11;821-8.

14 Claessens P, Moons P, Dierckx de Casterlé B, et al. What does it mean to live with a congenital heart disease? A qualitative study on the lived experiences of adult patients. Eur J Cardiovasc Nursing 2005:4:3-10.

15 Knowles RL, Bull C, Wren C, et al. Ethics, governance and consent in the UK: implications for research into the longer-term outcomes of congenital heart defects. Arch Dis Child 2011:96:14-20. 